Check for updates

Cite this: RSC Adv., 2017, 7, 24647

\title{
Self-assembled chiral phosphorus nanotubes from phosphorene: a molecular dynamics study $\uparrow$
}

\begin{abstract}
Douxing Pan, (D) abc Tzu-Chiang Wang, ${ }^{* b}$ Chao Wang, ${ }^{b}$ Wei Guo a and Yugui Yao*a
Controlled syntheses in nanoscale structures should be expected and phosphorous nanotubes with predefined chiralities are important in electronic devices with tunable bandgap. Here, incorporating molecular dynamics simulations with theoretical analyses, we show that a zigzag phosphorene nanoribbon can self-assemble and form a corresponding chiral phosphorous nanotube surrounding a template armchair phosphorous nanotube. The van der Waals potential between the nanoribbon and the nanotube is transformed to the intrinsic deformed and chemical bonding energies of the synthesized tube together with partial kinetic energy. The self-assembly process has an apparent temperature dependence and size effect and the formed chiral tube is thermodynamically stable. Also, the chirality and measurement can be tuned by the radius of template tube and the aspect ratio of raw ribbon. The study suggests a novel and feasible approach for controlled synthesis of phosphorous nanotubes and thus is of great interest for semiconductor device applications.
\end{abstract}

Received 3rd April 2017

Accepted 1st May 2017

DOI: 10.1039/c7ra03807k

rsc.li/rsc-advances

desired for electronic devices with tunable bandgap. On the other hand, due to the realization of graphene with tailored properties using different techniques, ${ }^{13-16}$ self-assemblies of graphene ribbons (GNRs) have been extensively investigated, theoretically and experimentally., ${ }^{2,11-13}$ Also, with the everincreasing controllabilities in supramolecular organizations, ${ }^{2,12,14,15}$ GNRs have been proposed for the novel generation of nanostructures including carbon nanoscrolls (CNSs), ${ }^{2,17,18}$ twisted GNRs and chiral CNTs, ${ }^{12,15}$ as well as versatile configurations assembled by fullerenes and graphene flakes including disordered, parallel and vertical wrappings. ${ }^{19}$ However, can the self-assembly happen for double-atomically thin phosphorene? If yes, can chiral phosphorus nanotubes ( $\mathrm{cPNTs})^{20}$ be assembled from phosphorene with tailored shapes and sizes, and how?

In this work, we demonstrate by classic molecular dynamics (MD) simulations the self-assembly mechanical behaviors of phosphorene nanoribbons (PNRs) on the surface of PNTs. The study shows that an armchair phosphorous nanotube (aPNT) can induce a zigzag phosphorene nanoribbon (zPNR) to self-assemble into a corresponding cPNT. The van der Waals (vdW) interaction between the aPNT and zPNR is the driving force of self-assembly behavior. The selfassembly is not only dependent on the radius of template tube and the size of raw ribbon, but also on the temperature. The formed chiral tube is thermodynamically stable and its chirality can be well controlled theoretically. Our study provides a novel and feasible approach for the fine controlled synthesis of phosphorene allotropes and novel phosphorenebased composite functional nanostructures, and should be of great importance to developments of electronic devices with tunable bandgap.
${ }^{a}$ Laboratory of Quantum Functional Material Design and Application, School of Physics, Beijing Institute of Technology, Beijing 100081, China. E-mail: ygyao@bit. edu.cn.

${ }^{b}$ State Key Laboratory of Nonlinear Mechanics, Institute of Mechanics, Chinese Academy of Sciences, Beijing 100190,China.E-mail: tcwang@imech.ac.cn 'University of Chinese Academy of Sciences, Beijing 100190, China

$\dagger$ Electronic supplementary information (ESI) available. See DOI: 10.1039/c7ra03807k 
The computational model is shown in Fig. 1, consisting of a zigzag phosphorene nanoribbon with a length $L_{x}$, width $L_{y}$ and thickness $h$ and a template armchair phosphorus nanotube with an equivalent radius $r$. The aPNT with a length at least $2 L_{y}$, is initially fixed at a distance of $1.5 r$ away from left side of the zPNR and around $0.35 \mathrm{~nm}$ above the zPNR along $z$-direction which is the equilibrium distance between the aPNT and zPNR. Three different aPNTs with radii $r=1.10,1.47$ and $1.84 \mathrm{~nm}$, are used to explore the curvature of the template aPNT on the selfassembly behavior of the zPNR. Similar to the slenderness ratio of the column for characterization of size effect in mechanics of materials, ${ }^{21}$ an aspect ratio of the zPNR is introduced as

$$
\delta=\frac{L_{x}}{L_{y}}=\frac{m\left|\boldsymbol{a}_{2}\right|}{n\left|\boldsymbol{a}_{1}\right|}=\alpha \frac{m}{n}
$$

where lattice vectors $\boldsymbol{a}_{1}$ and $\boldsymbol{a}_{2}$ are along $\operatorname{armchair}(n, 0)$ and zigzag $(0, m)$ directions of phosphorene, respectively, and $n, m$ are number of unit cells along the two typical directions, as denoted in Fig. 1. $\alpha=\left|\boldsymbol{a}_{2}\right| /\left|\boldsymbol{a}_{1}\right|$ is a dimensionless parameter and can be viewed as $\alpha=1$ for convenience.

All simulations were carried out within the framework of classic MD-based method, implemented in the large-scale atomic/molecular massively parallel simulator (LAMMPS) code. $^{22}$ The interatomic interactions in aPNT or zPNR are characterized by the Stillinger-Weber (SW) potential, ${ }^{23}$ which has been previously parameterized to correctly describe the mechanical and thermal properties of phosphorene by Jiang et $a l .{ }^{24}$ and $\mathrm{Xu}$ et $a l .,{ }^{25}$ and cited by the literatures mentioned in the introduction. ${ }^{6,9,10}$ The interaction between aPNT and zPNR is described by the Lennard-Jones (LJ) potential

$$
V_{\mathrm{LJ}}(r)=4 \varepsilon\left[(\sigma / r)^{12}-(\sigma / r)^{6}\right]
$$

where $\varepsilon=0.0159 \mathrm{eV}$ and $\sigma=0.3438 \mathrm{~nm}$ for bilayer phosphorene. ${ }^{26}$

Prior to simulations, the initial aPNT and zPNR equilibrium configurations were both achieved via the conjugate-gradient (CG) algorithm. Then, the aPNT-zPNR system under free boundary conditions was relaxed to reach a stable thermodynamic state in a canonical (NVT) ensemble with a time step of 1 fs for $\sim 0.5 \mathrm{~ns}$ in Nose-Hoover thermostat. As done in other works, ${ }^{2,6,9,10,14}$ for facilitating the discussion, particularly, for

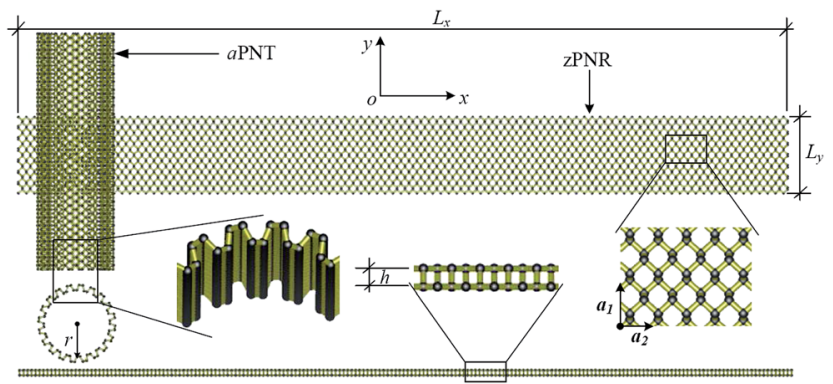

Fig. 1 Computational model of zPNR and aPNT. $L_{x}, L_{y}$ and $h$ are length, width and thickness of zPNR, respectively, and $r$ is an inbetween radius of aPNT. Lattice vectors $a_{1}$ and $a_{2}$ are along armchair $(n, 0)$ and zigzag $(0, m)$ directions, respectively. focusing on the physical and mechanical nature of the "chiral" self-assembly itself, we presuppose that experiments are performed in vacuum or inert atmosphere to avoid terminating the edges of zPNR due to ambient oxygen or humidity in practice. ${ }^{27}$

Fig. 2 shows snapshots at four successive stages of the selfassembly process of a zPNR with aspect ratio $\delta=13.86$ on a fixed aPNT at a constant environmental temperature $30 \mathrm{~K}$. Initially, the whole zPNR is at an activated state accompanying slightly bending, twisting, shrinking and expanding due to the thermal motion at a finite environmental temperature as shown in Fig. 2a. As the ribbon sticking the fixed tube, its left side starts to slide and rolls on the outside of the nanotube due to the vdW interaction from the aPNT with the remaining part keeping swinging as shown in Fig. 2b. Interestingly, the nano-ribbon can rearrange itself from the initial state with the longitudinal direction perpendicular to the axis of aPNT as shown in Fig. 1 to form a spiral helix angle and starts to roll around the nano-tube, which is much different from the self-assembly of GNR. ${ }^{12,14,15}$ At the same time, the partly-rolled phosphorene ribbon slides much quickly and reciprocally along the tube as the rolling process. A new perfect PNT with certain chirality (which has been actually formed at the second stage shown in Fig. 2b under the given condition) is formed gradually due to the coalescence of connectors as more part of the ribbon is rolled on the aPNT as shown in Fig. 2c and d. The whole process has been videotaped as Movie1.avi in our ESI, $\dagger$ which illustrates the dynamics better than structural snap shots.

The above thermodynamic self-assembly is driven by the minimization of free energy as shown in Fig. 3a, where the (external) total, potential and vdW energy are depicted against simulation time. All these energies decrease gradually with respect to time and reach a stable minimum (at $t_{0} \sim 0.16 \mathrm{~ns}$ as

(a)

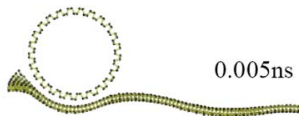

(b)

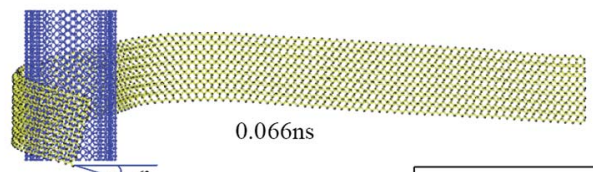

(c)

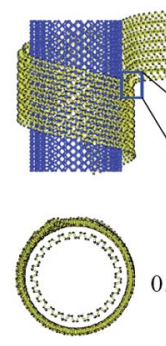

(e)

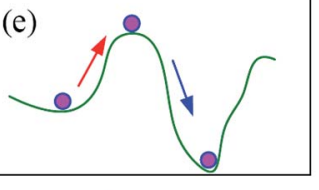

(d)

Fig. 2 Thermodynamic process. Snapshots of self-assembly process of $z \operatorname{PNR}(\delta=13.86)$ on aPNT( $n=25)$ at four different moments from (a) to (d) (see also Movie1.avi†). Chiral angle $\varphi$ in (b) is formed gradually at this stage. The enlarged part in (c) displays bonding process in zPNR edges squeezed each other. (e) Schematic diagram for van der Waals ( $v d W$ ) force induced local thermal energy overcoming energy barriers from (intrinsic) Stillinger-Weber(SW) potential. 

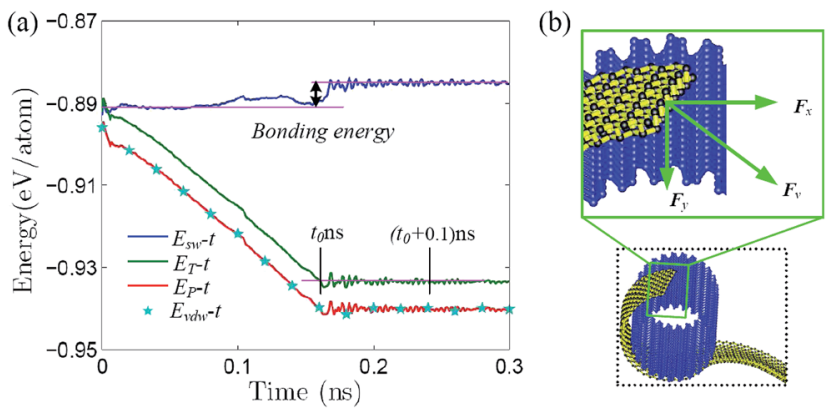

Fig. 3 Energy feature and free-body diagram. (a) Variation of the (SW/ total/potential/vdW) energy with respect to simulating time for the thermodynamic process of aPNT( $n=25)$ interacting with $\mathrm{zPNR}(\delta=$ 13.86). Stable (external) total energy level is reached at $t_{0}$ ns and a stable chiral tube can be extracted from the simulating system at $\left(t_{0}+0.1\right) \mathrm{ns}$. (b) Free-body diagram for nanoribbon rolled on the outer wall of template tube.

denoted in the figure for the total energy), which is similar to that of GNR..$^{2,12,14,15}$ The free energy of zPNR self-assembly after equilibrium is much larger than that of GNR, ${ }^{14-16}$ since the configuration energy of graphene is $-9.214 \mathrm{eV}$ per atom, much smaller than that of phosphorene, $-5.365 \mathrm{eV}$ per atom, according to density functional theory (DFT) calculations., ${ }^{3,5,22}$ The potential curve is coincided with that of the vdW interaction indicating that the self-assembly process is actually driven by the vdW force. Fig. 3b presents a free-body diagram for the part of raw ribbon rolled on the surface of template tube and the traction force $F_{\mathrm{v}}$ acting on the nano-ribbon from vdW interaction, which can be decomposed into the component $F_{\mathrm{x}}$ at the tangent of nano-tube pulling the ribbon to roll around the ring, and the component $F_{\mathrm{y}}$ along the axis of nano-tube pulling the ribbon to slide along the groove of corrugated crystal.

The chiral vector of the chiral phosphorus nanotube generated can be described theoretically as

$$
\boldsymbol{C}_{\mathrm{h}}=n \boldsymbol{a}_{1}+m_{\mathrm{c}} \boldsymbol{a}_{2}=n \boldsymbol{a}_{1}+\left[\frac{\sqrt{\left(\pi d_{\mathrm{c}}\right)^{2}-\left(n\left|\boldsymbol{a}_{1}\right|\right)^{2}}}{\left|\boldsymbol{a}_{2}\right|}\right] \boldsymbol{a}_{2}
$$

where $d_{\mathrm{c}}=2(r+h+\sigma)$ is diameter of $\operatorname{cPNT}\left(n, m_{\mathrm{c}}\right)$ and $[x]$ denotes nearest integer of $x$. Note that more precise lattice constants based on the DFT results $\left|\boldsymbol{a}_{1}\right|=0.462 \mathrm{~nm}$ and $\left|\boldsymbol{a}_{2}\right|=0.330 \mathrm{~nm}$ are adopted for convenience of theoretical predictions, ${ }^{5}$ which are in agreement with that obtained from our MD calculations (the average lattice constants are $0.441 \mathrm{~nm}$ and $0.331 \mathrm{~nm}$, respectively). According to eqn (3), the chiral angle in Fig. 2 b can be written as

$$
\varphi=\frac{180}{\pi} \arctan \left[\left(n\left|\boldsymbol{a}_{1}\right|\right) /\left(m_{\mathrm{c}}\left|\boldsymbol{a}_{2}\right|\right)\right]
$$

We conducted a series of simulations using zPNRs with varied aspect ratios $\delta$ and aPNTs with different radii $r$ or chiral indexes $n$ to compare the theoretical predictions with MD simulations in which stable chiral tubes can be extracted from the simulating system at $\left(t_{0}+0.1\right) \mathrm{ns}$. It is shown from Table 1 that the predictions of chiralities for the produced phosphorus
Table 1 Comparisons between theoretical formulas and MD simulation for the results of the chiralities from self-assembled cPNT by zPNR on $\mathrm{aPNT}^{a}$

\begin{tabular}{llll}
\hline & \multicolumn{2}{c}{ cPNT $\left[\left(n, m_{\mathrm{c}}\right) \mid\left(\varphi /^{\circ}\right)\right]$} & \\
\cline { 2 - 3 } $\begin{array}{l}\text { zPNR }\left(L_{y} / \mathrm{nm}\right) \\
h=0.21 \mathrm{~nm}]\end{array}$ & Formula $(3) \mid(4)$ & MD-simulation & $\begin{array}{l}\text { aPNT }(r / \mathrm{nm}) \\
{[\sigma=0.35 \mathrm{~nm}]}\end{array}$ \\
\hline $1.84(\delta=24.25)$ & $(4,31) \mid 10.24$ & $(4,30) \mid 10.58$ & $1.10\left(N_{\mathrm{L}}=30\right)$ \\
$1.84(\delta=48.25)$ & $(4,45) \mid 7.10$ & $(4,45) \mid 7.10$ & $1.84\left(N_{\mathrm{L}}=30\right)$ \\
$3.23(\delta=20.71)$ & $(7,37) \mid 14.84$ & $(7,37) \mid 14.84$ & $1.47\left(N_{\mathrm{L}}=30\right)$ \\
$3.23(\delta=20.71)$ & $(7,44) \mid 12.56$ & $(7,44) \mid 12.56$ & $1.84\left(N_{\mathrm{L}}=20\right)$ \\
$3.23(\delta=13.86)$ & $(7,44) \mid 12.56$ & $(7,44) \mid 12.56$ & $1.84\left(N_{\mathrm{L}}=30\right)$
\end{tabular}

${ }^{a}$ Note that: $\sigma=0.35 \mathrm{~nm}$ is an average relaxed distance between phosphorene ribbon and phosphorus nanotube, slightly different from that for bilayer phosphorene.

nanotubes by eqn (3) and (4) are well consistent with the results of MD simulations, meaning that the chirality of the selfassembled cPNT is dependent on the width $L_{y}=n\left|\boldsymbol{a}_{1}\right|$ of zPNR and the radius $r$ of aPNT.

Fig. 4 presents three typical cPNTs with front and top views, whose radii $r_{\mathrm{c}}$ are 1.66, 2.02, $2.39 \mathrm{~nm}$, respectively. The enlarged part in Fig. 4a as an example displays the perfect bonding structure in $\operatorname{cPNT}(4,30)$. Such a mutual bonding was also discussed by Czerwinski, et al. based on classic AIREBO force field $^{28}$ and so was it for Tersoff potential in some specific conditions (such as high pressure). ${ }^{21,22,29}$ But in current case, it is not only attributed to the dangling chemical bonds in zPNR edges, but also due to the strong constraining force from vdW interaction overcoming the activation energy barriers from intrinsic SW potential. ${ }^{21,23}$ Fig. 3e presents a schematic diagram, where vdW force induced local thermal energy makes the bonding edges (interaction) climb to a high energy barrier of SW potential, and then drop to a lower energy well. Therefore, as just shown in the detailed illustration of Fig. 2c, the helical pitch becomes so small that the zPNR edges can squeeze and bond with each other. It should be noted that, the strong impact can be, essentially, attributed to an extremely high pressure, and the bonding just forms by means of the mathematical form of non-mechanical (SW) potential functions consisting of twoand three-body interactions. ${ }^{21-23,29}$ It is also noted that some mechanical classical force fields such as CHARMM, can not arrive at bond breaking or forming even in current case, where bonds, angles and dihedrals are all assigned according to the (phosphorene) crystal configuration. ${ }^{\mathbf{1 4 2 1 , 2 2}}$ Moreover, Fig. 3a presents a variation of SW energy against the simulation time. It can be seen that the energy curve almost hold a level value before the third stage and after the forth stage of the winding process except tiny fluctuations due to small local distortions of the zPNR. A general increasing energy is observed from $\sim 0.09$ to $\sim 0.16 \mathrm{~ns}$ with evident fluctuations. It shows that an average bonding energy is saved in the new tube as an intrinsic SW potential during the bonding process, accompanying by large local distortions. The single bond forming can be roughly estimated as a local thermal energy at extremely high temperature of $29158 \mathrm{~K}\left(k_{\mathrm{B}} T=0.005 \mathrm{eV}\right.$ per atom in Fig. 3a with total 5025 atoms in the system and assuming that 10 atoms form 
(a)
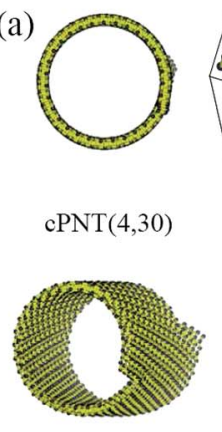

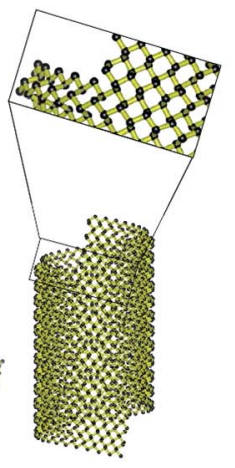

(b)
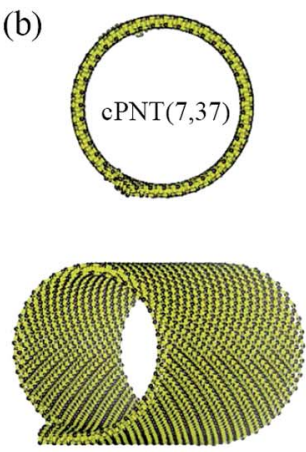

(c)

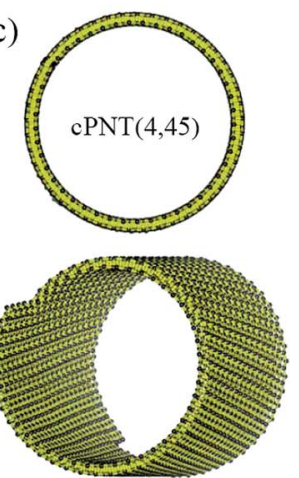

Fig. 4 Three typical cPNTs with isometric and top views: (a) CPNT(4, 30), (b) cPNT(7, 37) with a Movie2.avi presented in the ESI, $\uparrow$ (c) cPNT(4, 45), whose self-assemblies are induced by the aPNTs with $n=15,20$ and 25, respectively. The enlarged part in (a) displays the bonding structure in $\operatorname{cPNT}(4,30)$.

chemical bonds at the same time). This further demonstrates a fully admissible bonding condition. Comparing $\operatorname{cPNT}(4,30)$ in Fig. 4a with $\operatorname{cPNT}(4,45)$ in Fig. $4 \mathrm{c}$ for the same chirality index $n$ $=4$, it is known that the chirality of son-tube is independent on the length $L_{\mathrm{a}}$ (also, $N_{\mathrm{L}}=L_{\mathrm{a}} /\left|\boldsymbol{a}_{2}\right|$ as an integer indicator listed in Table 1) of parent-tube and/or the length $L_{x}$ of raw-ribbon, but its length can be measured by them fully, and could approximately conform to the theoretical formula

$$
L_{\mathrm{c}} \approx\left(\frac{m}{m_{\mathrm{c}}}-1\right) n\left|\boldsymbol{a}_{2}\right| \sin \left(\frac{\varphi \pi}{180}\right)
$$

For the simulated $\operatorname{cPNT}(7,44)$ in Table 1 with the same width $L_{y}=3.23 \mathrm{~nm}$ of zPNRs and the same radius $r=1.84 \mathrm{~nm}$ of the aPNT, according to eqn (5) the effective lengths $L_{\mathrm{c}}$ are calculated as $0.60 \mathrm{~nm}$ and $1.15 \mathrm{~nm}$, respectively, both in good agreement with the results of MD simulations.

Due to the anisotropic nature of phosphorene and the bending instability along its zigzag direction, ${ }^{5,10}$ the zPNRs prefer moving forward in a spiral way outside the aPNTs to bend themselves directly, and finally form chiral tubes with different radii, as shown in Fig. 4a-c. Recently, Fernández-Escamilla et al. pointed out based on their DFT calculations that cPNTs hold larger binding energy than zPNTs and the values are comparable to aPNTs. ${ }^{20}$ For checking the stability of assembled cPNT, the ESI Movie2.avi $\uparrow$ presented the thermodynamic process of $\operatorname{cPNT}(7,37)$ shown in Fig. $4 \mathrm{~b}$ at $100 \mathrm{~K}$ and the asymmetric ends was directly chopped off (e.g., a recent controlled sculpture method of phosphorene nanoribbons developed by Das et al. ${ }^{30}$ ) to eliminate the sway induced the stress concentration. The result shows that the chiral tube can hold at least $0.4 \mathrm{~ns}$ at $100 \mathrm{~K}$ in a canonical ensemble, much more stable than the zPNT. ${ }^{10}$ Also, the large thermal motion of the $\operatorname{cPNT}(7,37)$ is constrained by the SW potential to be a corresponding thermal deformation and partially heat-driven oscillations.

In order to further study the temperature dependence and size effect, it is necessary to introduce a self-assembly energy $E_{\text {es }}$ and velocity $V_{\text {es }}$ as

$$
E_{\mathrm{es}}=E_{\mathrm{s}}-E_{\mathrm{e}} \text { and } V_{\mathrm{es}}=\frac{L_{x}}{t_{\mathrm{e}}-t_{\mathrm{s}}}
$$

where $E_{\mathrm{s}}$ and $E_{\mathrm{e}}$ are initial and final total energy, and $t_{\mathrm{s}}$ and $t_{\mathrm{e}}$ indicate inital and final time of the self-assembly with $t_{\mathrm{s}}=0$ and $t_{\mathrm{s}}=t_{0}+0.1$, as noted in Fig. 1a. The investigated temperature is kept at $T<100 \mathrm{~K}$ or one third of room temperature during simulations, which can be viewed as an intermediate temperature condition to avoid instability of the formed chiral tube as stated above; $;^{6,9,10}$ meanwhile, too low temperature is not allowed owing to no enough kinetic energy moving the raw ribbon and pushing it sticking the template tube.
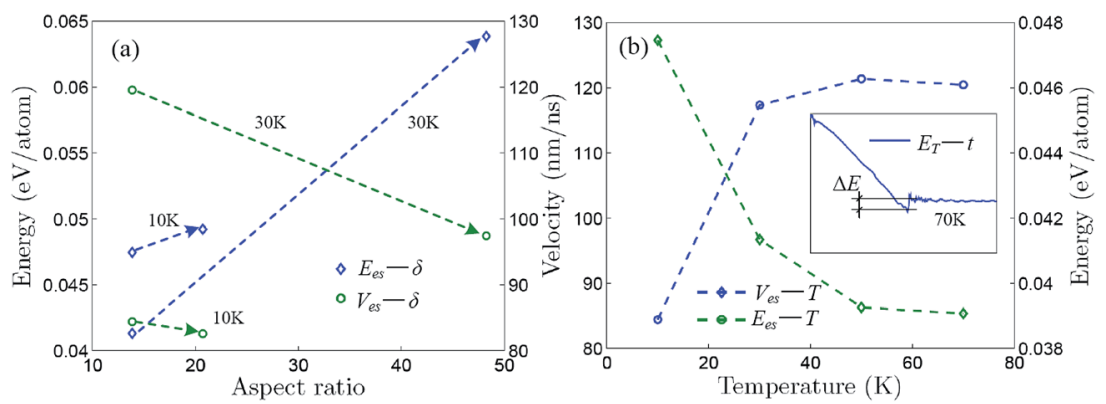

Fig. 5 Self-assembly energy and velocity curves. Self-assembly energy and velocity change with (a) aspect ratio for self-assembly induced by $\operatorname{aPNT}(n=25)$ and (b) Kelvin temperature for self-assembly of zPNT( $\delta=13.86)$ induced by aPNT( $n=25)$. The inset in (b) shows variation of total energy with respect to time at $70 \mathrm{~K}$, in which a fracture energy $\Delta E$ is defined approximately, being the same as the average bonding forming energy presented in Fig. 3a. 
Fig. 5a shows that the self-assembly energy and velocity change with aspect ratio for the self-assemblies induced by the $\operatorname{aPNT}(n=25)$ and the self-assembly energy increases while the self-assembly velocity decreases with aspect ratio, regardless of the temperature being at 10 or $30 \mathrm{~K}$. Moreover, for the aPNT $(n=$ $25)$ induced the self-assembly of the $\operatorname{zPNR}(\delta=13.86)$, as presented in Fig. 5b, the self-assembly energy decreases while the self-assembly velocity increases with temperature. This is because the higher temperature mainly contributes the kinetic energy and makes the average atom velocity quicker to speed up the self-assembly process. Under given temperature and length of one side, the smaller aspect ratio mainly lowers the potential energy difference shortening the self-assembly time and makes the rolling easier on the outside surface of aPNT. ${ }^{\mathbf{1 4 2 1}}$ At the temperature of $70 \mathrm{~K}$ or higher, the self-assembly process becomes so fierce that a fracture behavior happens, as shown in the inset of Fig. $5 \mathrm{~b}$ for details, and this further demonstrates the controlled temperature should be kept at $T<100 \mathrm{~K}$.

In summary, a self-assembly mechanical behavior from phosphorene nanoribbon to chiral phosphorous nanotube was studied systematically for the first time, based on classic molecular dynamics simulations incorporating theoretical analysis. We predicted that a template armchair phosphorus nanotube can induce a zigzag phosphorene nanoribbon to form a corresponding chiral phosphorous nanotube. The van der Waals interaction between the template nanotube and raw nanoribbon is the driving force of self-assembly process, which leads to the bending, twisting and shrinking of the raw ribbon in vacuum and sticking, sliding and rolling outside the template tube. Particularly, the interaction is transformed to the intrinsic deformed and chemical bonding energies of the synthesized tube after overcoming the energy barriers from Stillinger-Weber potential. A positive correlation was found between the self-assembly energy and aspect ratio or between the self-assembly velocity and temperature, while a negative correlation was found between the self-assembly energy and temperature or between the self-assembly velocity and aspect ratio. The formed chiral tube is thermodynamically stable and its chiralities and sizes can be controlled pretty well.

\section{Acknowledgements}

The work reported here is supported by the NSFC of China (Grant No. 11602270, 11574029, and 11532013), the MOST of China (Grant No. 2014CB920903, 2016YFA0300600), and the National Basic Research Program of China ("973" Project, Grant No. 2012CB937500). The first author thanks Dr Dongmei Hu in Jilin University for helpful discussions and the reviewer from ACS Nano for his/her constructive suggestions on classic bonding mechanism.

\section{References}

1 J. A. Rogers, M. G. Lagally and R. G. Nuzzo, Nature, 2011, 477, 45 .
2 X. H. Shi, N. M. Pugno and H. J. Gao, J. Comput. Theor. Nanosci., 2010, 7, 1.

3 L. Li, et al., Nat. Nanotechnol., 2014, 9, 372.

4 A. A. Kistanov, Y. Q. Cai, K. Zhou, S. V. Dmitriev and Y.-W. Zhang, J. Phys. Chem. C, 2016, 120, 6876.

5 D. X. Pan, Y. Li, T.-C. Wang and W. Guo, Acta Mech. Sin., 2017, 33, 71.

6 Z. Y. Yang, J. H. Zhao and N. Wei, Appl. Phys. Lett., 2015, 107, 023107.

7 Y. Ding, Y. Wang, L. Shi, Z. Xu and J. Ni, Phys. Status Solidi $R R L, 2014,8,939$.

8 J.-W. Jiang and H. S. Park, Nat. Commun., 2014, 5, 4727.

9 Z.-D. Sha, Q.-X. Pei, Z. W. Ding, J.-W. Jiang and Y.-W. Zhang, J. Phys. D: Appl. Phys., 2015, 48, 395303.

10 X. B. Liao, F. Hao, H. Xiao and X. Chen, Nanotechnology, 2016, 27, 215701.

11 A. V. Talyzin, I. V. Anoshkin, A. V. Krasheninnikov, R. M. Nieminen, A. G. Nasibulin, H. Jiang and E. I. Kauppinen, Nano Lett., 2011, 11, 4352.

12 O. O. Kit, T. Tallinen, L. Mahadevan, J. Timonen and P. Koskinen, Phys. Rev. B: Condens. Matter Mater. Phys., 2012, 85, 085428.

13 H. E. Lim, Y. Miyata, R. Kitaura, Y. Nishimura, Y. Nishimoto, S. Irle, J. H. Warner, H. Kataura and H. Shinohara, Nat. Commun., 2013, 4, 2548.

14 T.-C. Wang, C. Zhang and S. H. Chen, J. Nanosci. Nanotechnol., 2013, 13, 1136.

15 C. Zhang, Z. L. Peng and S. H. Chen, J. Phys. Chem. C, 2014, 118, 19477.

16 Y. Wang, H. F. Zhan, C. Yang, Y. Xiang and Y. Y. Zhang, Comput. Mater. Sci., 2015, 96, 300.

17 D. Yu and F. Liu, Nano Lett., 2007, 7, 3046.

18 L. He, J.-Q. Lu and H. Jiang, Small, 2009, 5, 2802.

19 J.-W. Feng, H.-M. Ding and Y.-Q. Ma, Carbon, 2015, 90, 34.

20 H. N. Fernández-Escamilla, J. J. Quijano-Briones and

A. Tlahuice-Flores, Phys. Chem. Chem. Phys., 2016, 18, 12414.

21 J. Morrison, Modern Physics for Scientists and Engineers, Academic Press, 2nd edn, 2015.

22 S. Plimpton, J. Comput. Phys., 1996, 117, 1.

23 F. H. Stillinger and T. A. Weber, Phys. Rev. B: Condens. Matter Mater. Phys., 1985, 31, 5262.

24 J.-W. Jiang, T. Rabczuk and H. S. Park, Nanoscale, 2015, 7, 6059.

25 W. Xu, L. Y. Zhu, Y. Q. Cai, G. Zhang and B. W. Li, J. Appl. Phys., 2015, 117, 214308.

26 J.-W. Jiang and H. S. Park, J. Appl. Phys., 2015, 117, 124304. 27 R. A. Doganov, et al., Nat. Commun., 2015, 6, 6647.

28 B. Czerwinski, Z. Postawa, B. J. Garrison and A. Delcorte, Nucl. Instrum. Methods Phys. Res., Sect. B, 2013, 303, 22.

29 J. Tersoff, Phys. Rev. B: Condens. Matter Mater. Phys., 1998, 37, 6991.

30 P. M. Das, et al., ACS Nano, 2016, 10, 5687. 